\title{
Activation and enhancement of caerulomycin A biosynthesis in marine-derived Actinoalloteichus sp. AHMU CJ021 by combinatorial genome mining strategies
}

Yunchang Xie', Jiawen Chen ${ }^{1}$, Bo Wang ${ }^{3,4}$, Tai Chen ${ }^{3,4}$, Junyu Chen ${ }^{1}$, Yuan Zhang ${ }^{2}$, Xiaoying Liu²* and Qi Chen ${ }^{2^{*}}$ (1)

\begin{abstract}
Background: Activation of silent biosynthetic gene clusters (BGCs) in marine-derived actinomycete strains is a feasible strategy to discover bioactive natural products. Actinoalloteichus sp. AHMU CJ021, isolated from the seashore, was shown to contain an intact but silent caerulomycin A (CRM A) BGC-cam in its genome. Thus, a genome mining work was preformed to activate the strain's production of CRM A, an immunosuppressive drug lead with diverse bioactivities.

Results: To well activate the expression of cam, ribosome engineering was adopted to treat the wild type Actinoalloteichus sp. AHMU CJ021. The initial mutant strain XC-11 G with gentamycin resistance and CRM A production titer of $42.51 \pm 4.22 \mathrm{mg} / \mathrm{L}$ was selected from all generated mutant strains by gene expression comparison of the essential biosynthetic gene-camE. The titer of CRM A production was then improved by two strain breeding methods via UV mutagenesis and cofactor engineering-directed increase of intracellular riboflavin, which finally generated the optimal mutant strain XC-11GUR with a CRM A production titer of $113.91 \pm 7.58 \mathrm{mg} / \mathrm{L}$. Subsequently, this titer of strain XC-11GUR was improved to $618.61 \pm 16.29 \mathrm{mg} / \mathrm{L}$ through medium optimization together with further adjustment derived from response surface methodology. In terms of this 14.6 folds increase in the titer of CRM A compared to the initial value, strain XC-GUR could be a well alternative strain for CRM A development.
\end{abstract}

Conclusions: Our results had constructed an ideal CRM A producer. More importantly, our efforts also had demonstrated the effectiveness of abovementioned combinatorial strategies, which is applicable to the genome mining of bioactive natural products from abundant actinomycetes strains.

Keywords: Caerulomycin A, Genome mining, Combinatorial strategies, Ribosome engineering, Strain improvement, Marine-derived Actinoalloteichus

*Correspondence: liuxiaoying@ahmu.edu.cn; chenqi@ahmu.edu.cn ${ }^{2}$ School of Life Sciences, Anhui Medical University, Hefei 230032, China Full list of author information is available at the end of the article

\section{Background}

Actinomycetes, the acknowledged producers of bioactive secondary metabolites, are historically important bioresources in the discovery and development of pharmaceutical molecules [1-5]. In particular, marine actinomycetes

c) The Author(s) 2020. This article is licensed under a Creative Commons Attribution 4.0 International License, which permits use, sharing, adaptation, distribution and reproduction in any medium or format, as long as you give appropriate credit to the original author(s) and the source, provide a link to the Creative Commons licence, and indicate if changes were made. The images or other third party material in this article are included in the article's Creative Commons licence, unless indicated otherwise in a credit line to the material. If material is not included in the article's Creative Commons licence and your intended use is not permitted by statutory regulation or exceeds the permitted use, you will need to obtain permission directly from the copyright holder. To view a copy of this licence, visit http://creativeco mmons.org/licenses/by/4.0/. The Creative Commons Public Domain Dedication waiver (http://creativecommons.org/publicdomain/ zero/1.0/) applies to the data made available in this article, unless otherwise stated in a credit line to the data. 
thriving under the extreme environmental conditions are different from their terrestrial counterparts, which enables marine-derived strains to acquire excellent characteristics for the biosynthesis of numerous bioactive secondary metabolites $[6,7]$. Thus, marine actinomycetes are becoming the emerging resources for exploring novel drug leads $[8,9]$.

However, the production of bioactive secondary metabolites in marine actinomycetes is always hampered due to unavailable expression of their biosynthetic gene clusters (BGCs) under laboratory conditions $[8,10,11]$. To unlock the biosynthesis potential of these strains, the innovative genome mining strategy-ribosome engineering is adopted to activate the expression of essential biosynthetic genes of cryptic secondary metabolites in strain's genome [12-18]. Ribosome engineering can usually introduce mutations into the bacterial RNA polymerase $\beta$-subunit or ribosomal protein $\mathrm{S} 12$ by using rifampicin or streptomycin, respectively $[14,16,17]$. These induced mutations can modulate gene expression to activate biosynthesis of secondary metabolites, leading to discover bioactive natural products from marine actinomycetes $[19,20]$.

Caerulomycin A (CRM A), a new marine immunosuppressive agent with diverse bioactivities, was isolated from the marine-derived actinomycete Actinoalloteichus sp. 2216-6 [21]. The biosynthesis of this marine drug lead is initiated by the formation of a core $2,2^{\prime}$-dipyridine skeleton, which is catalyzed by a unique hybrid polyketide-nonribosomal peptide (PKS-NRPS) assembly line [22-26]. Then, the 2,2'-dipyridine skeleton is finally converted into the CRM A by a series of post-modification reactions, including amino hydrolysis, oxime formation and methylation [23, 27-30]. CRM A can induce the generation of regulatory $\mathrm{T}$ cells to avoid $\mathrm{T}$ cell responses and change the function of B cells, which significantly inhibits the Mixed Lymphocyte Reaction (MLR) [31, 32]. These prominent bioactivities enable CRM A to be a promising immunosuppressive drug lead, and it has been used in prolonging the survival of allogeneic skin grafts [33].

Given the pharmaceutical prospect of CRM A, screening more original marine strains and solving the yield limitation problems could be an efficient approach in its future development. Actinoalloteichus sp. AHMU CJ021 was a new offshore sediment-derived actinomycetes strain. The subsequent sequencing analysis confirmed an intact BGC responsible for CRM A biosynthesis was located in the strain's genome. However, this strain could not produce CRM A because its BGC was always silent in laboratory fermentations.

Thus, to activate the biosynthesis of CRM A in Actinoalloteichus sp. AHMU CJ021, a gentamycinresistant mutant strain $\mathrm{XC}-11 \mathrm{G}$ was obtained by gene expression-directed ribosome engineering. This strain, unlike the rifamycin or streptomycin-induced mutant strains, did not have definite mutation sites but could produce CRM A with an initial titer of $42.51 \pm 4.22 \mathrm{mg} / \mathrm{L}$. Then this titer was rapidly enhanced to $618.61 \pm 16.29 \mathrm{mg} / \mathrm{L}(\approx 14.6$ folds $)$ in a following mutant strain XC-11GUR by the subsequent combinatorial methods: UV mutagenesis, cofactor engineeringdirected riboflavin supplement optimization, medium optimization integrated with response surface methodology-oriented adjustment. In conclusion, our study had finally woken up the silent gene cluster cam and significantly enhanced the CRM A production in Actinoalloteichus sp. AHMU CJ021. This reconstruction of new CRM A producer also established the combinatorial strategies composed of strain breeding methods (ribosome engineering, UV mutagenesis and genetic engineering) and medium optimization, which could be effectively utilized in genome mining bioactive natural products and efficient producing strains.

\section{Results \\ Genomic analysis of the CRM A biosynthetic potential in Actinoalloteichus sp. AHMU CJ021}

Actinoalloteichus sp. AHMU CJ021 was isolated from the marine sediment. Considering the unique survival environment and secondary metabolite production potential of this strain, the whole genome scanning was adopted, and a 6,825,770 bp genome (NCBI accession number: CP025990) with an average of $72.31 \% \mathrm{G}+\mathrm{C}$ content was obtained (Fig. 1 and Additional file 1: Tables S1 and S2). Subsequent secondary metabolite biosynthesis potential assessments revealed that approximately 22 BGCs were distributed in the strain genome (Fig. 1 and Additional file 1: Table S3). Among these BGCs, the core region of cluster 3, named cam (37.9 kb length, from $245,751 \mathrm{bp}$ to $283,656 \mathrm{bp}$ ), shows $95 \%$ high similarity to two well-identified CRM A BGCs: crm (Actinoalloteichus cyanogriseus WH1-2216-6) and cae (Actinoalloteichus cyanogriseus NRRL B-2194) [23, 26] (Fig. 2). The cam contains 20 ORFs including essential dipyridine ring biosynthetic genes and backbone post-modification genes [23, 26, 28-30] (Fig. 2 and Additional file 1: Table S4), which indicates that Actinoalloteichus sp. AHMU CJ021 can biosynthesize CRM A with the same classic mechanism as that of the two aforementioned phylogenetically identified homologous strains (Fig. 3a and Additional file 1: Fig. S1).

\section{Activation of CRM A biosynthesis by gene expression-directed ribosome engineering}

Given the high similarity in all three BGCs, Actinoalloteichus sp. AHMU CJ021 was fermented to produce CRM A by inoculating the strain into previously used 


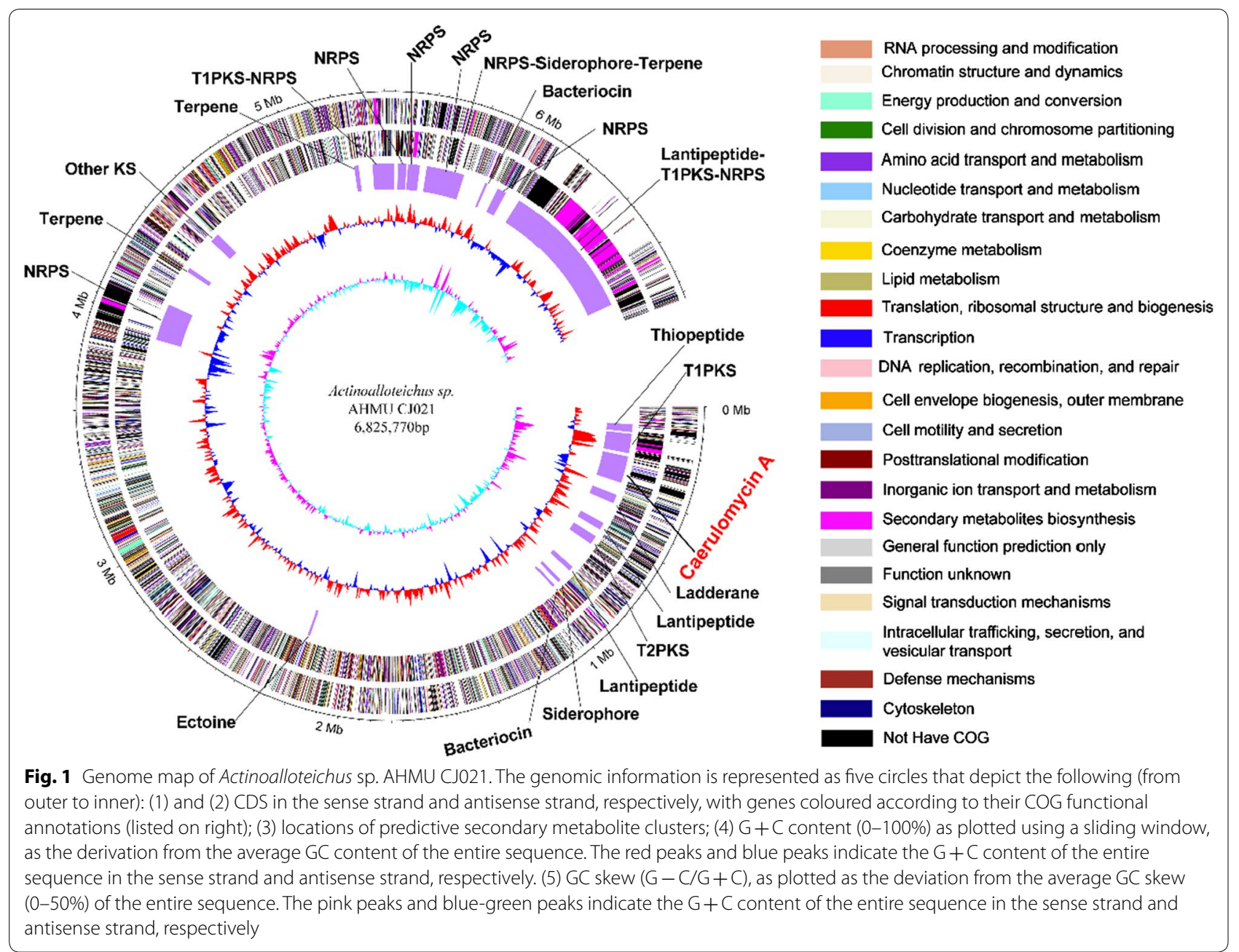

media (Additional file 1: Table S5) or other laboratory media. Unexpectedly, no CRM A was detected from the fermentation extracts (Fig. 3b). Further RT-PCR analysis revealed that the essential biosynthetic gene camE responsible for CRM A backbone assembly was not expressed in the abovementioned media (Fig. 3c, d). These gene expression results indicated that cam was silent under routine conditions.

To activate the expression of cam, Actinoalloteichus sp. AHMU CJ021 was subjected to ribosome engineering treatment. Hence, a series of 17 mutants were generated by three antibiotics according to the protocols in the methods section (Additional file 1: Table S6). Then, three camE-expressing mutants were further selected by the RT-PCR assay (Fig. 3c). The optimal mutant strain XC-11G with $30 \mu \mathrm{g} / \mathrm{mL}$ gentamicin resistance $(2 \times \mathrm{MIC})$ and the highest $c a m E$ expression level was finally selected after qPCR analysis (Fig. $3 \mathrm{~d}$ and Additional file 1: Table S7). Subsequent fermentation and HPLC analysis obviously revealed that strain XC-11G could produce a new compound (Fig. 3b lane ii). To our delight, this compound was identified as CRM A by ${ }^{1} \mathrm{H}$ NMR, ${ }^{13} \mathrm{C}$ NMR and HR-ESI-MS(+) (Additional file 1: Figs. S2, S3, S4 and Additional file 1: Table S8), which verified that cam expression and CRM A biosynthesis were effectively activated. Subsequent measurements further confirmed that the strain XC-11G produced CRM A with a titer of $42.51 \pm 4.22 \mathrm{mg} / \mathrm{L}$ (Additional file 1: Table S7).

The genetic characterization of rps12 (NCBI accession number: WP_016698050.1), rpl6 (NCBI accession number: WP_026419518.1) and 16S rDNA (NCBI accession number: CP025990; 1,052,752-1,054,270) from $\mathrm{XC}-11 \mathrm{G}$ revealed that no clear mutation site was located, which was consistent with previously reported cases [34-37]. Additionally, the genetic stability of $\mathrm{XC}-11 \mathrm{G}$ in CRM A biosynthesis was then validated by successive fermentation comparisons among three generations from XC-11G (Additional file 1: Fig. S5a). 


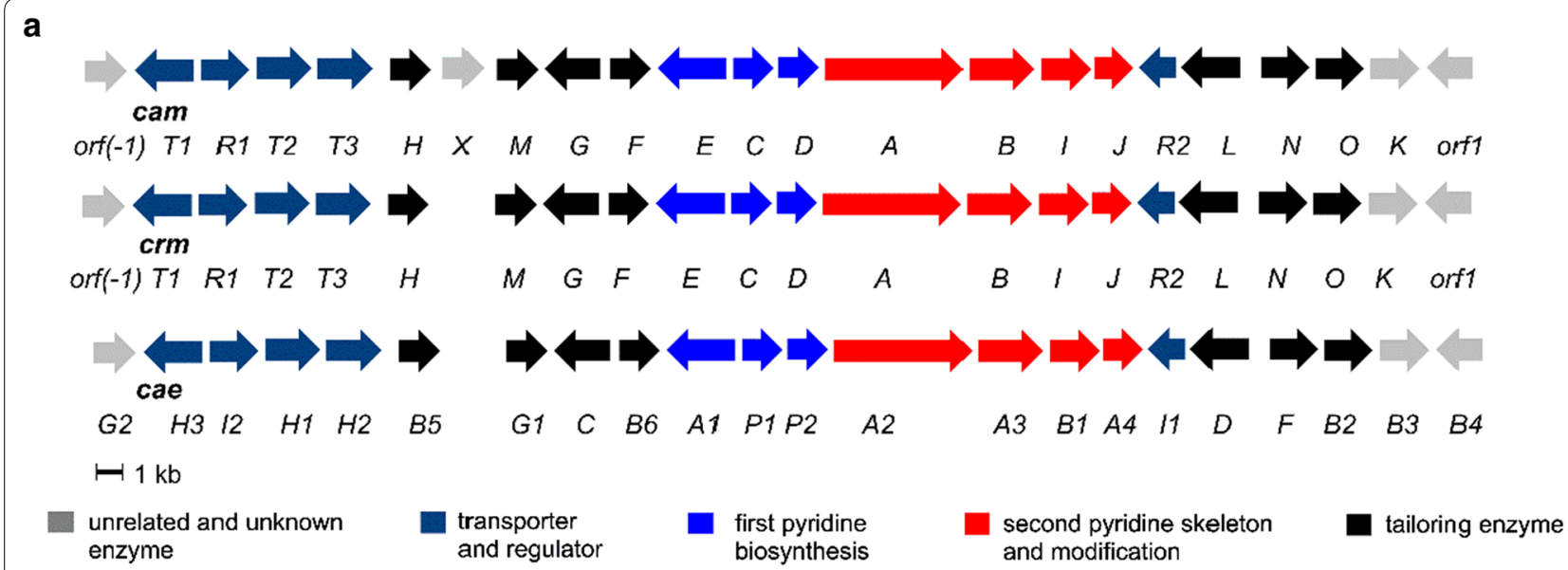

b

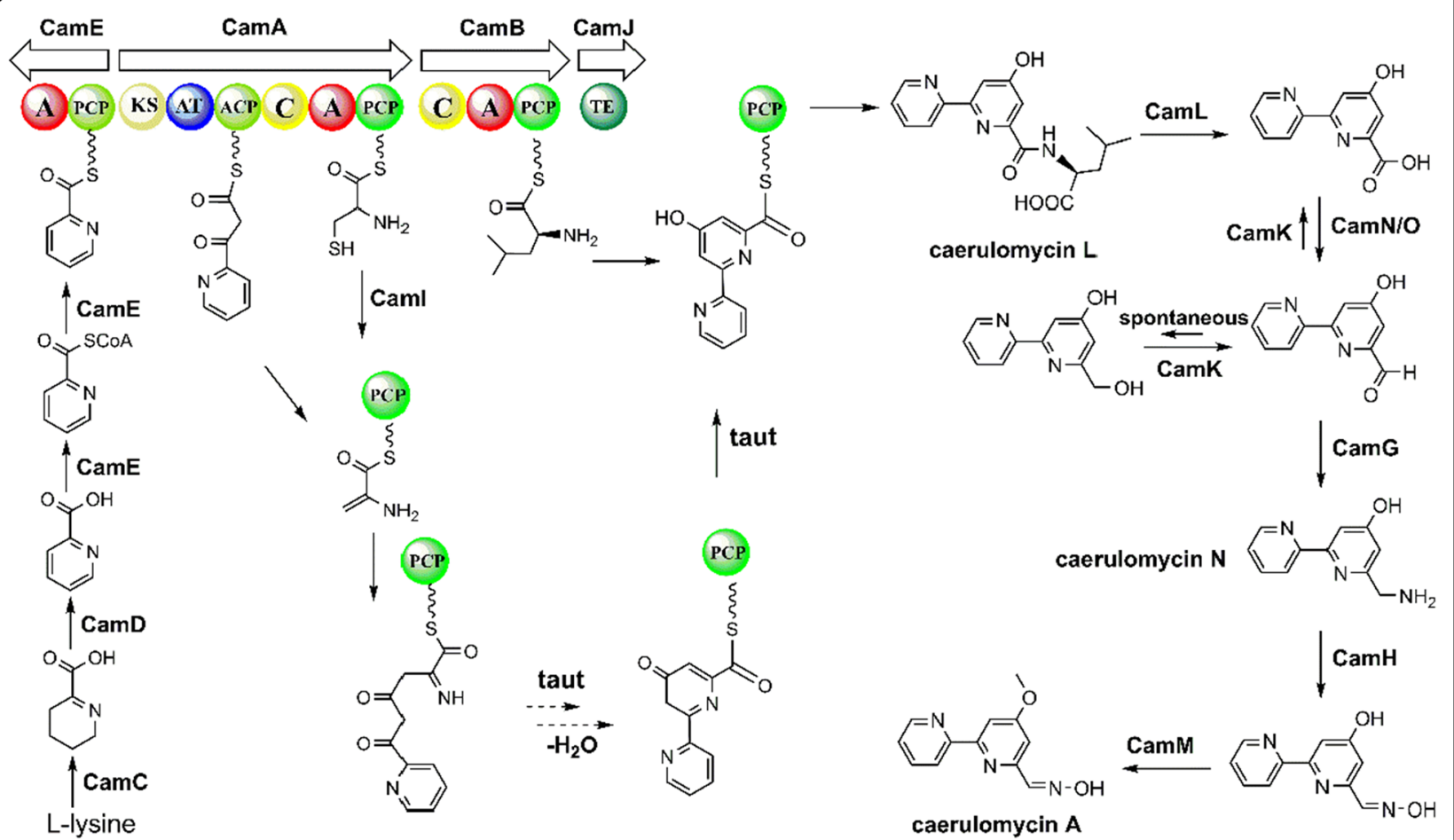

Fig. 2 a Genetic organization of the CRM A biosynthetic gene clusters in Actinoalloteichus sp. AHMU CJ021 (cam), A. cyanogriseus WH1-2216-6 (crm) and Actinoalloteichus cyanogriseus NRRL B-2194 (cae). b The proposed model for caerulomycin A biosynthesis in Actinoalloteichus sp. AHMU CJ021. A: adenylation domain; C: condensation; ACP: acyl carrier protein; PCP: peptidyl carrier protein; AT: acyltransferase; KS: $\beta$-ketoacyl-ACP synthase; TE: thioesterase

Enhancement of CRM A production by UV mutagenesis To improve CRM A production efficiently, the UV mutagenesis (traditional high mutation rate method) was adopted to treat the strain XC-11G. After UV irradiation, 105 mutants (over 99.99\% lethal rate) were recovered for the following tube-scale fermentation and bioactivity assays of the corresponding extracts. Through comparison of the inhibition zones between UV mutant strains and the preliminary XC-G11 (inhibition zone approximately $16-22 \mathrm{~mm}), 4$ mutants with improved antibacterial bioactivity (inhibition zone $>22 \mathrm{~mm}$ ) were screened for subsequent fermentation validation (Fig. 3e). The mutant strain XC-11GU was selected among these 4 mutant strains for its highest CRM A yield titer of 
$78.62 \pm 3.55 \mathrm{mg} / \mathrm{L}$, with an approximately $85 \%$ increase compared to that of the preliminary XC-11G (Fig. 3b lane iii and Additional file 1: Table S9). Furthermore, the stability of this improvement in CRM A biosynthesis of XC$11 \mathrm{GU}$ was validated by the same fermentation process of evaluating its three successive generations (Additional file 1: Fig. S5b).

\section{Further enhancement of CRM A production by optimizing intracellular riboflavin supplement}

Enhancing the cofactor level by engineering its biosynthetic process could drive metabolic flux to improve the biosynthesis of target metabolites [38]. This new metabolic engineering strategy named as cofactor engineering has been well applied to microbial second metabolites development [39-41]. Previous study had revealed that the biosynthesis of CRM A required several essential flavoenzymes, including CamD, which completed the formation of the picolinic acid precursor; CamH, which catalyzed the formation of the oxime group, and CamK, which maintained the substrate recycling process $[23,28,29]$. Thus, enhancing the intracellular riboflavin supplement could facilitate the formation of these flavoenzymes, which may accelerate CRM A production in turn. As is wellknown, the riboflavin biosynthesis is controlled by the catalytic efficiency of type II guanosine triphosphate (GTP) cyclohydrolase (GCH II) [42, 43]. Accordingly, in situ overexpression of this rate-limiting GCH II encoded gene could improve intracellular riboflavin biosynthesis [42], which may provide increased amounts of flavin cofactor into the secondary metabolite biosynthetic pathway. This method has been well utilized in Nocardiopsis flavescens CGMCC 4.5723, another marine-derived rare actinomycetes strain, to enhance its production of marinacarboline A [44]. Based on the similarity, the in situ overexpression of AsribA (NCBI accession number AUS77540.1), encoding GCH II in Actinoalloteichus sp. AHMU CJ021, was implemented to construct a new mutant strain
XC-11GUR. Although no obvious growth and morphological changes in XC-11GUR were noted relative to the two aforementioned mutant strains, $\mathrm{XC}-11 \mathrm{G}$ and $\mathrm{XC}-11 \mathrm{GU}$ (Fig. 3f), the riboflavin production titer of XC-11GUR steadily increased to $0.51 \pm 0.03 \mathrm{mg} / \mathrm{L}$, with a $35 \%$ increase compared with that of the wild type strain and the two other mutant strains (Fig. 3g). Importantly, the expected steady enhancement of CRM A production, with a titer of $113.91 \pm 7.58 \mathrm{mg} / \mathrm{L}$ and an approximately $45 \%$ increase compared with that of the previous strain $\mathrm{XC}-11 \mathrm{GU}$, was also achieved in the new optimal mutant strain XC-11GUR and its three successive generations (Fig. $3 \mathrm{~b}$ lane iv and Additional file 1: Fig. S5c).

\section{Medium optimization for CRM A production}

The abovementioned mutant strains were screened and fermented on ISP2 medium, which is convenient for large-batch strain production yield comparison due to its simple but nutritious composition. However, the medium is an essential element in actinomycete secondary metabolites development. Given this important role of the medium, several well-utilized media (Medium 1-7) and two new media (Medium N1-N2) were adopted for CRM A production comparison in mutant strain XC-11GUR fermentation (Additional file 1: Table S5). Among them, the Medium N2, first designed and used in this study, gave rise to the best yield titer of $238.65 \pm 3.14 \mathrm{mg} / \mathrm{L}$, which was approximately $1.2-7.6$ folds higher than that with the other media (Fig. 4a and Additional file 1: Table S10).

To further improve the fermentation on Medium N2, the significant variables of its in strains' CRM A production were screened through Plackett-Burman design (PBD). All nine ingredients of medium N2 with addition of the three dummy variables $\left(X_{d v 1}-X_{d v 3}\right)$ were designed as two-level factors for 20 tests (Additional file 1: Tables S11 and S12). After triplicate fermentation comparison, the average CRM A production (Additional file 1: Table S11) was subjected to an effect estimate process

\footnotetext{
(See figure on next page.)

Fig. 3 a Phylogenetic analysis of Actinoalloteichus sp. AHMU CJ021. Numbers at the nodes represent bootstrap percentages obtained from 1000 replicates. The scale bar (0.005) represents nucleotide substitutions per site. Sequence alignment was performed with ClustalW. A phylogenetic tree was constructed with MEGA 7.0 software using neighbor-joining algorithm based on $16 \mathrm{~S}$ rDNA gene sequences; $\mathbf{b}$ HPLC analyses of CRM A production (asterisk) in Actinoalloteichus sp. AHMU CJ021 wild type and mutant strains. (i) Actinoalloteichus sp. AHMU CJ021 wild type; (ii-iv) XC-11G, XC-11GU and XC-11GUR respectively; Transcriptional analysis, c RT-PCR assay and $\mathbf{d}$ QPCR assay, determined camE expression in the Actinoalloteichus sp. AHMU CJ021 wild type strain (WT) and three mutants: XC-16R (M1), XC-14G (M2) and XC-11G (M3); e The bioassay comparison and screening of mutants generated by UV mutagenesis, the asterisk indicates the inhibition zone level of the original mutant XC-11G; the number of mutants belonging to each range is indicated up the column; f The cultured morphology of the Actinoalloteichus sp. AHMU CJ021 wild type strain and three mutants on M-ISP2 medium; $\mathbf{g}$ HPLC analyses for riboflavin (black solid circle) biosynthesis in the Actinoalloteichus sp. AHMU CJ021 wild type and mutant strains. (i) authentic standard of riboflavin; (ii) Actinoalloteichus sp. AHMU CJ021 wild type; (iii-v) XC-11G, XC-11GU and XC-11GUR, respectively
} 

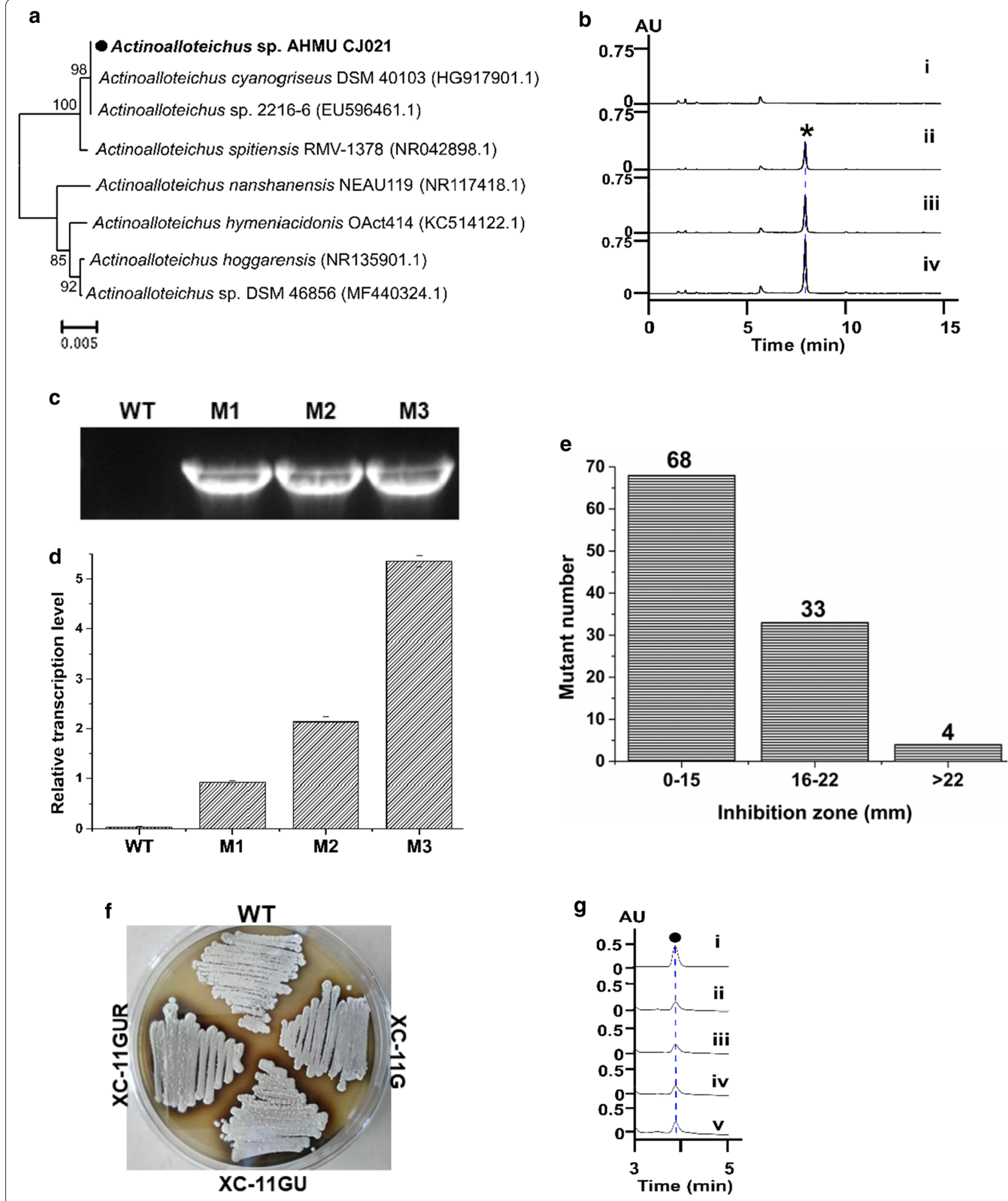

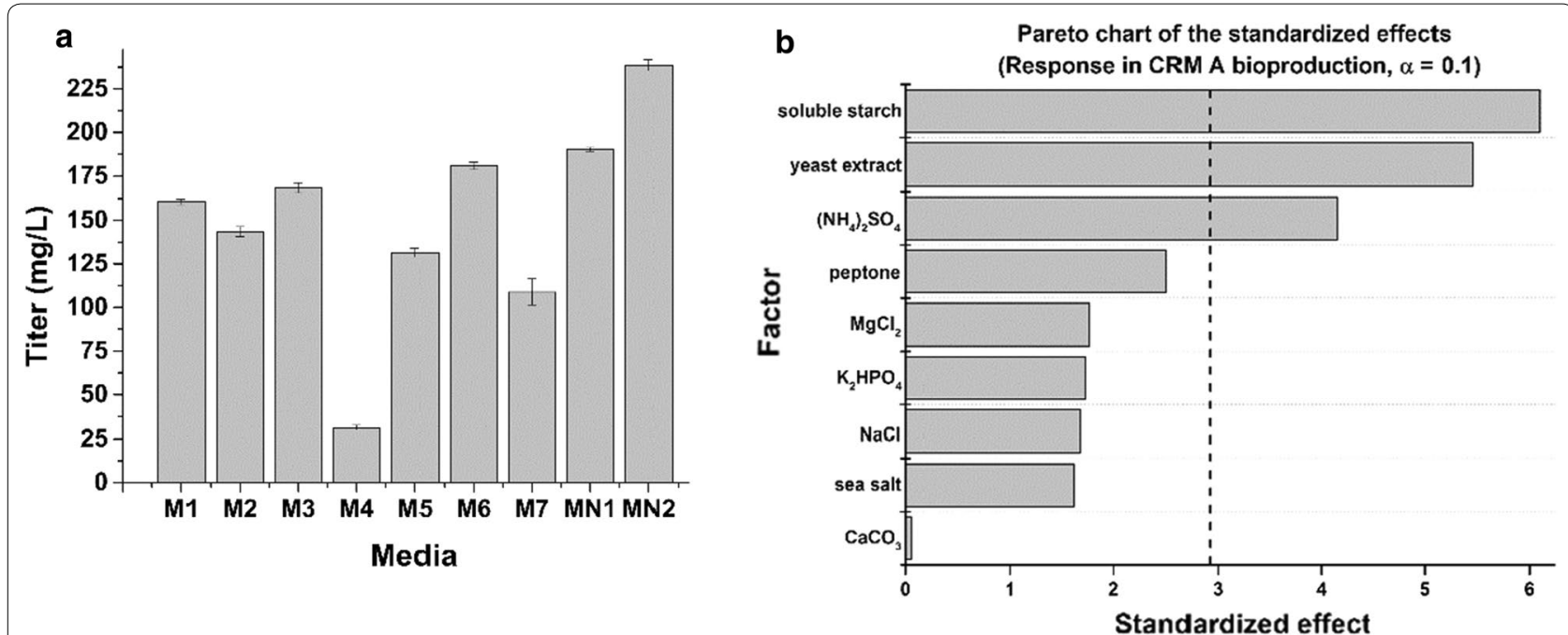

Fig. 4 a The CRM A production titer on different media (Additional file 1:Table S1); 1-7 represent the Medium 1-Medium 7 (Additional file 1: Table S1); N1 represents the Medium N1 and N2 represents the Medium N2 (Additional file 1: Table S2); b Pareto chart displays the standardized effects of each variable and indicates three most significant factors of medium N2 for improving the CRM A production titer. The bars of the diagram that go beyond the vertical dashed line ( $95 \%$ confidence level) correspond to the statistically significant standardized effects

(Additional file 1: Table S13). Based on these analyses, three factors, including soluble starch $\left(X_{1}\right)$, yeast extract $\left(X_{2}\right)$ and $\left(\mathrm{NH}_{4}\right)_{2} \mathrm{SO}_{4}\left(X_{5}\right)$, obviously showed above $95 \%$ high confidence level $(p<0.05)$ in the Pareto chart analysis (Fig. 4b). Therefore, these three factors were the critical significant variables of medium N2 optimization to improve CRM A production.

\section{Further optimization of CRM A production by using response surface methodology}

To enhance the CRM A production of strain XC-11GUR on medium N2 to a better level, a Box-Behnken design (BBD) type response surface analysis was employed to determine the optimal concentration of the three variables verified above. After experimental design and fermentation tests (Additional file 1: Tables S14 and S15), the corresponding results and data were subjected to multiple regression analysis. Finally, the equation describing the relationship between CRM A titer $(Y)$ and the necessary three variables (soluble $\operatorname{starch} / X_{1}$, yeast extract $/ X_{2}$ and $\left.\left(\mathrm{NH}_{4}\right)_{2} \mathrm{SO}_{4} / X_{5}\right)$ was confirmed as follow:

$$
\begin{aligned}
Y= & 572.72-80.09 X_{1}+71.51 X_{2}-58.55 X_{5}-59.29 X_{1}^{2} \\
& -56.39 X_{2}^{2}-1.8 X_{1} X_{2}-41.4 X_{1} X_{5}-10.19 X_{2} X_{5}
\end{aligned}
$$

Additionally, the reliability of the above-obtained model was tested as highly significant by analysis of variance (ANOVA) of CRM A titer to draw high $F$-value and low $p$ value $(0.00$ or $<0.05)$ in regression coefficient validation (Table 1). Meanwhile, another coefficient estimate showed that most regression coefficients were also highly
Table 1 Analysis of variance (ANOVA) for the second-order polynomial model

\begin{tabular}{lcrrr}
\hline Source & $\begin{array}{l}\text { Degree } \\
\text { of freedom }\end{array}$ & Sum of squares & $\boldsymbol{F}$ value & $\boldsymbol{p}$ value \\
\hline Model & 9 & 147,256 & 163.16 & 0.000 \\
Linear & 3 & 119,650 & 397.71 & 0.000 \\
Quadratic & 3 & 25,786 & 85.71 & 0.000 \\
Cross product & 3 & 1821 & 6.05 & 0.041 \\
Residual & 5 & 501 & & \\
Lack of fit & 3 & 239 & 0.61 & 0.672 \\
Pure error & 2 & 263 & & \\
Total & 14 & 147,758 & & \\
\hline
\end{tabular}

Table 2 The least-squares fit and coefficient estimate

\begin{tabular}{lclrl}
\hline Variables & $\begin{array}{l}\text { Coefficient } \\
\text { estimate }\end{array}$ & Standard error & $\boldsymbol{t}$ value & $\boldsymbol{p}$ value \\
\hline Intercept & 572.72 & 5.78 & 99.06 & 0.000 \\
$X_{1}$ & -80.09 & 3.54 & -22.62 & 0.000 \\
$X_{2}$ & 71.51 & 3.54 & 20.20 & 0.000 \\
$X_{5}$ & -58.55 & 3.54 & -16.54 & 0.000 \\
$X_{1}^{2}$ & -59.29 & 5.21 & -11.38 & 0.000 \\
$X_{2}^{2}$ & -56.39 & 5.21 & -10.82 & 0.000 \\
$X_{5}^{2}$ & -35.80 & 5.21 & -6.87 & 0.001 \\
$X_{1} X_{2}$ & -1.80 & 5.01 & -0.18 & 0.864 \\
$X_{1} X_{5}$ & -41.40 & 5.01 & -4.13 & 0.009 \\
$X_{2} X_{5}$ & -10.19 & 5.01 & -1.02 & 0.356 \\
\hline
\end{tabular}



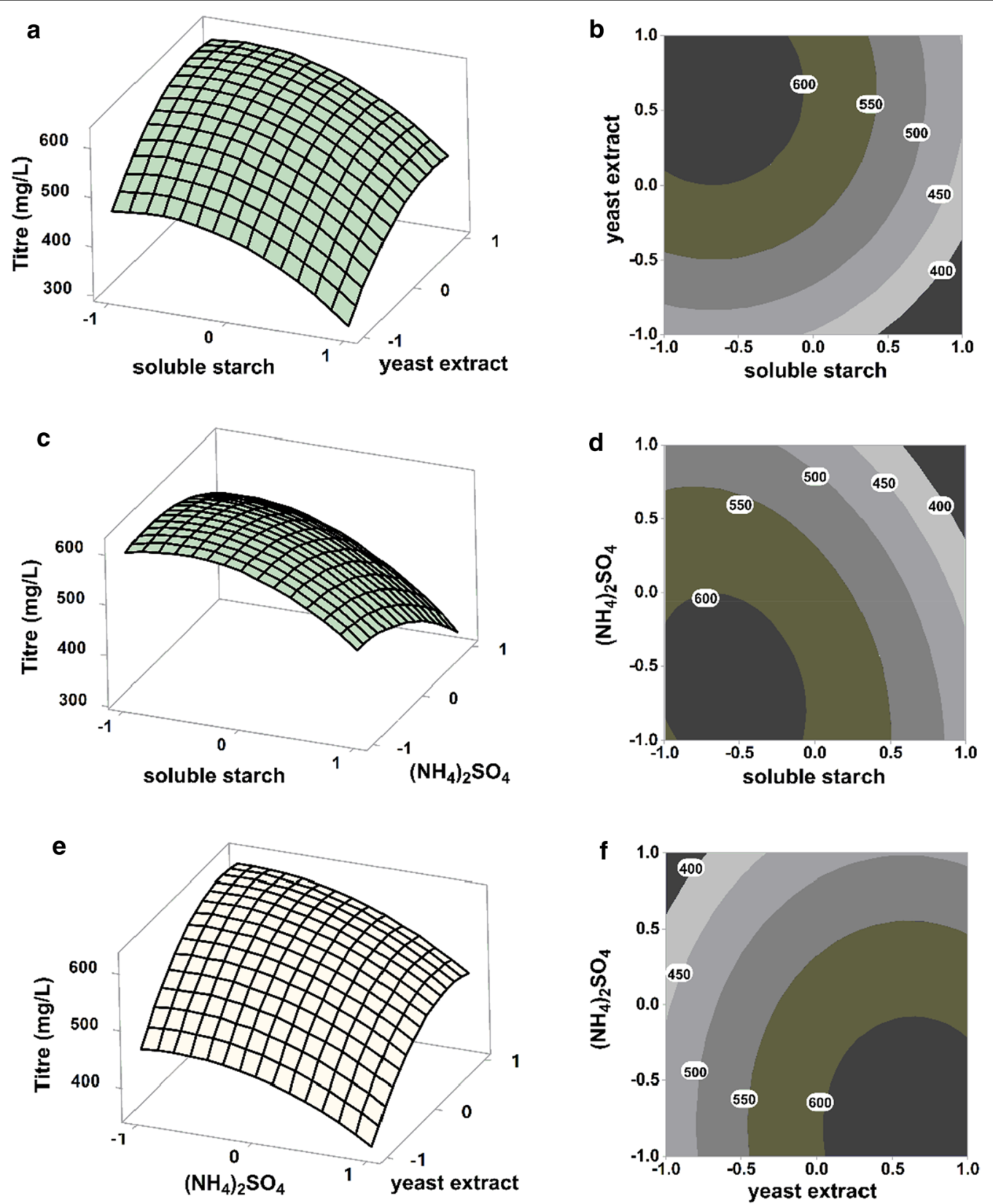

Fig. 5 The 3D response surfaces and 2D contour plots for CRM A production in Medium N2. The effect of two variables on CRM A production is presented in each figure, while the other variable is held at zero level. $\mathbf{a}, \mathbf{b}$ The effects of soluble starch $\left(X_{1}\right)$ and yeast extract $\left(X_{2}\right)$; $\mathbf{c}, \mathbf{d}$ The effects of soluble starch $\left(X_{1}\right)$ and $\left(\mathrm{NH}_{4}\right)_{2} \mathrm{SO}_{4}\left(X_{5}\right)$; e, $\mathbf{f}$ The effects of yeast extract $\left(X_{2}\right)$ and $\left(\mathrm{NH}_{4}\right)_{2} \mathrm{SO}_{4}\left(X_{5}\right)$

significant for the low $p$-value $(<0.05)$. Furthermore, the consistency relevant to two coefficients of determinations: $R^{2}(99.66 \%)$ and adjusted $R^{2}(99.05 \%)$, reflected the ideal fit between the observed and predicted responses (Table 2). These agreements once again confirmed present model to be reliable for CRM A production.

The graphical regression equation, displayed on the $3 \mathrm{D}$ response surfaces and 2D contour plots (Fig. 5), obviously reflected the optimum selection of three related variables or factors for CRM A titer in the maximal response. Clearly, the CRM A titer improved with increasing concentration of yeast extract $/ X_{2}$ (level $1 \sim 0.7$ ), but with decreasing concentrations of soluble starch $/ X_{1}$ (level $\left.1 \sim-0.6\right)$ and $\left(\mathrm{NH}_{4}\right)_{2} \mathrm{SO}_{4} / X_{5}$ (level $1 \sim-0.7)$. The maximum yield of CRM A based on the optimized ingredient content $\left(X_{1}\right.$ level $=-0.556$, 

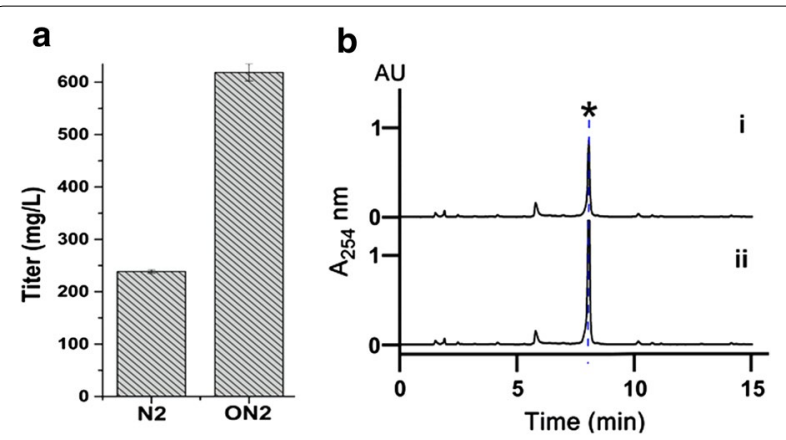

Fig. 6 a The CRM A production titer of mutant XC-11GUR on Medium N2 (N2) and optimized medium N2 (ON2). b The HPLC analysis of CRM A production (asterisk) of mutant XC-11GUR on Medium N2 (i) and Optimized Medium N2 (ii)

$10.67 \mathrm{~g} / \mathrm{L}$ soluble starch; $X_{2}$ level $=0.677,3.83 \mathrm{~g} / \mathrm{L}$ yeast extract; $X_{3}$ level $\left.=-0.679,1.00 \mathrm{~g} / \mathrm{L}\left(\mathrm{NH}_{4}\right)_{2} \mathrm{SO}_{4}\right)$ was calculated as $639.63 \mathrm{mg} / \mathrm{L}$. To validate this theoretical production, a triplicate confirmatory fermentation with the optimized conditions were performed. As expected, a higher CRM A titer of $618.61 \pm 16.29 \mathrm{mg} / \mathrm{L}$ was obtained and agreed well with the predicted yield (Fig. 6).

\section{Discussion}

The activation of silent or cryptic secondary metabolite BGCs is an emerging approach in natural product discovery and drug lead development. Antibiotic-induced ribosome engineering is an effective strategy to achieve this goal of activating abundant dormant genes, which in turn fully exploits the potential and valuable bioresources embedded in strain. In the present study, the CRM A biosynthesis potential of marine-derived Actinoalloteichus sp. AHMU CJ021 was successfully activated by gentamycin-directed ribosome engineering. Meanwhile, this obtained mutant was also an estimable original strain for subsequent production improvement or further secondary metabolite discovery.

Unlike the well-characterized bioactivities of rifampicin and streptomycin to target bacterial RNA polymerase $\beta$-subunit and ribosomal protein S12, respectively, the mechanism of gentamycin-induced mutant is still unclear. The gentamycin-treated $E$. coli could be conferred mutations within 16S-rRNA [45], ribosomal protein S12 [46], or ribosomal protein L6 [47]. In contrast, this mechanism did not reappear in previously identified actinomycete mutants or the current mutant strain XC-11G derived from gentamycin-related ribosome engineering [33, 35, 36]. Although detailed mutation cases could not be clarified in the abovementioned mutant strain XC-11G, the productivity of CRM A is completely activated as a titer of $42.51 \pm 4.22 \mathrm{mg} / \mathrm{L}$. This upregulation of gene expression was due to the gentamycin-induced mutation similar to that of antibiotics used in ribosome engineering [12]. Maybe, mutation of the translation apparatus attacked by gentamycin was repaired in subsequent cultivation, which remained upregulated gene expression and drug resistance. These results unambiguously validated the feasibility and efficiency of gentamycininduced ribosome engineering, even if its detailed mechanism needed to be further investigated.

Significantly, recent progress in genomics and combinatorial biosynthesis research has identified many varied BGCs, providing sufficient authentic information to identify the necessary biosynthetic genes or gene clusters. Thus, the mutation process of ribosome engineering integrated with the expression comparison of essential biosynthetic genes could efficiently single out the desired high-yield and high-quality mutants without time or cost wasted on large-scale fermentation and bioactive comparison. In the present study, we selected camE (NCBI accession number: WP_103055331.1), with high similarity to well identified counterparts: $\mathrm{crmE}$ (NCBI accession number: AFD30957.1) and caeA1 (NCBI accession number: AFK24513.1) [23, 26], as the molecular marker to rapidly pick out the optimal mutant strain XC-11G from all 17 mutants based on camE expression differences. This approach avoided the meaningless and wasteful fullscale production verification or comparison.

Titer improvement is another key issue for production strain development. Traditional UV mutagenesis is a mature technique that induces a high mutation rate into the receptor strains. Engineering the riboflavin biosynthesis by increasing the supplement of the precursor to the flavin coenzyme formation is an efficient pathway that could indirectly activate the secondary metabolite biosynthesis [38-41]. This cofactor engineering type method has been well utilized in another marine actinomycete [44]. A straightforward strategy combining these two methods was applied for further production improvement of the original mutant strain XC-11G to obtain a more desirable mutant strain XC-11GUR, which effectively enhanced the CRM A production titer by approximately 2.7 folds increase. Not surprisingly, these methods combined with previous ribosome engineering could dramatically increase the ratio of positive results to efficiently enhance strain's CRM A productivity more than the inefficient using of any method alone.

As is known, the medium is another crucial condition for secondary metabolite production. In particular, the proper medium is always an influential factor in the expression of activated BGCs. Considering the important role of medium composition, the optimal mutant XC-11GUR was cultured 
with nine different media including six previously characterized CRM A production media, two new designed media, and ISP2 used as a convenient choice with simple but nutrient-rich ingredients (Additional file 1: Table S5). The generated CRM A yield varied significantly in these different media, and the optimum medium N2 was screened out, which had glucose (carbon source) removed but ammonium sulfate (inorganic nitrogen source) added. Glucose, a typical fast-acting carbon source, matching organic nitrogen sources is a common basic formula used in secondary metabolite production. However, supplementing some $\left(\mathrm{NH}_{4}\right)_{2} \mathrm{SO}_{4}$ as an inorganic nitrogen source and removing the commonly used glucose resulted in a significant enhancement in CRM A production. This empirical change provides a new CRM A titer or fermentation condition improvement option and once again confirms the importance of the medium in secondary metabolite production.

To further improve the medium N2, response surface methodology was adopted to determine the significant variables and further evaluate those effects, leading to the establishment of an optimized medium N2 composition (approximately 2.6 folds CRM A titer improvement) containing three critical factors were verified: $10.67 \mathrm{~g} / \mathrm{L}$ soluble

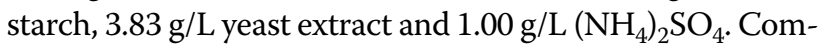
pared with that in the original medium N2, the amount of carbon source was further decreased. More notably, the ratio of yeast extract and peptone (organic nitrogen source) to $\left(\mathrm{NH}_{4}\right)_{2} \mathrm{SO}_{4}$ (inorganic nitrogen source) was increased from 2:1 (4-2 g/L) to approximately 6:1 (5.83-1 g/L), suggesting that the controlled carbon source feeding and high concentration of an organic nitrogen source coupled with supplementation of a limited inorganic nitrogen source could perfectly meet the requirements of CRM A biosynthesis to improve the CRM A yield in turn.

\section{Conclusion}

Overall, the CRM A production capacity of Actinoalloteichus sp. AHMU CJ021 was successfully activated and significantly improved by combining yield-oriented strain improvement via ribosome engineering, gene expression comparison integrated methods, UV mutagenesis, intracellular riboflavin biosynthesis enhancement, and empirical medium optimization coupled with response surface methodology-directed adjustment. Our efforts have obtained a new promising marine-derived CRM A high-yield producer that could be utilized for subsequent rational pathway engineering improvement. Moreover, our work also highlighted the efficacy of combinatorial strategies including empirical strain breeding methods and culture condition improvement approaches in rapid secondary metabolite improvement, which could facilitate discovery and development of natural products.

\section{Materials and methods}

Strain and culture conditions

Actinoalloteichus sp. AHMU CJ021 (the strain has been deposited to the China Center for Type Culture Collection with the number CCTCC M 2018157) was isolated from the $\sim 20 \mathrm{~cm}$ depth marine sediment on the seashore of Lianyungang, East China Sea. This strain was cultivated on solid ISP2 medium for sporulation. The selected fermentation media were described in Additional file 1: Table S5, which were also used to detect the CRM A production titer. For each fermentation, $50 \mu \mathrm{L}$ spore suspension $\left(10^{7}\right)$ of each strain was inoculated into $50 \mathrm{~mL}$ liquid fermentation medium supplied with $2 \%$ XDA- 16 resin in a $250 \mathrm{~mL}$ flask, then incubated at $28^{\circ} \mathrm{C}$ and $200 \mathrm{rpm}$ for 7 days.

\section{Whole genome scanning and sequence analysis}

The genomic DNA of Actinoalloteichus sp. AHMU CJ021 was extracted, sequenced and assembled completely by combining Illumina HiSeq2500 system and PacBio RSII high throughput sequencing technologies (BIOZERONShanghai; BGI-Shenzhen). Subsequently, functional genes were predicted by Glimmer and annotated by BlastP (https://blast.ncbi.nlm.nih.gov/Blast.cgi), referring to Swiss-Prot, COG and KEGG databases. The rRNA and tRNA genes were analyzed using RNAmmer and tRNAscan-SE, respectively.

Open reading frames (ORFs) were analyzed with the Frameplot 4.0 program (http://nocardia.nih.go.jp/fp4/) and the Blast program (http://blast.ncbi.nlm.nih.gov/). The PKS-NRPS domains were determined by web-based software (http://nrps.igs.umaryland.edu/nrps/).

The phylogenetic trees of $16 \mathrm{~S}$ rDNA between Actinoalloteichus sp. AHMU CJ021 and homologous strains were constructed with the Molecular Evolutionary Genetics Analysis (MEGA) 7.0 software using the neighbor-joining algorithm.

\section{Ribosome engineering of Actinoalloteichus sp. AHMU CJ021}

The minimal inhibition concentration of three commonly used antibiotics, namely, streptomycin, gentamycin, and rifamycin, against Actinoalloteichus sp. AHMU CJ021 was determined. Spore suspensions $\left(10^{6}-10^{7}\right.$ spores $)$ of this strain were spread onto ISP2 plates containing different concentrations of the three abovementioned antibiotics $(5-100 \mu \mathrm{g} / \mathrm{mL})$ and cultivated at $28{ }^{\circ} \mathrm{C}$ for persistent observation. The following treatments of the strains were carried out by applying the three abovementioned antibiotics at concentrations of $1 \times \mathrm{MIC}-5 \times \mathrm{MIC}$ on the ISP2 plates to obtain resistant mutant strains.

Genetic characterization of $30 \mathrm{~S}$ ribosomal protein S12 (NCBI accession number: WP_016698050.1), 
50S ribosomal protein L6 (NCBI accession number: WP_026419518.1) and 16S rDNA (NCBI accession number: CP025990; 1,052,752-1,054,270) of the final selected gentamycin-resistant mutant strains was performed by PCR using the primers listed in Additional file 1: Table S16. The amplified oligonucleotides were sequenced to verify the mutations (BGI-Shenzhen).

\section{Isolation and identification of CRM A from mutant strains}

To isolate CRM A from the mutant strains, a two-step fermentation process was adopted. First, a suitable portion of spores from a solid ISP2 medium plate was inoculated into $50 \mathrm{~mL}$ ISP2 medium and cultured at $28^{\circ} \mathrm{C}$ and $200 \mathrm{rpm}$ for $36 \mathrm{~h}$. Then, this seed culture $(50 \mathrm{~mL})$ was transferred into $200 \mathrm{~mL}$ ISP2 medium in a $1 \mathrm{~L}$ flask and cultured at $28{ }^{\circ} \mathrm{C}$ and $200 \mathrm{rpm}$ for an additional 6-7 days. Multiple flasks were used for repeating. Finally, the culture broth was centrifuged and divided into a supernatant and a mycelium cake. The supernatant was extracted by butanone at least two times and evaporated to dryness; the mycelium was extracted with acetone and evaporated to dryness too; Both organic extracts were dissolved in a 1:1 mixture of $\mathrm{CHCl}_{3} / \mathrm{MeOH}$ and mixed with an appropriate amount of silica gel for normal phase silica gel column chromatography, eluted with a successive gradient elution of $\mathrm{CHCl}_{3} / \mathrm{MeOH}$ mixture at $100 / 0$, 98/2, 96/4, 95/5, 93/7, 90/10, 80/20 and 50/50 to yield eight fractions ( $\mathrm{Fr} 1-\mathrm{Fr} 8)$. The fractions were analyzed in order by HPLC-UV. Fr3-Fr4 were subjected to another normal phase silica gel column chromatography step and eluted with a 100/0-0/100 gradient elution of a $\mathrm{ddH}_{2} \mathrm{O} /$ $\mathrm{MeOH}$ mixture to yield thirteen fractions (FrA1-FrA13). FrA5-FrA8 were evaporated to dryness and subjected to a PE/EtOH mixture (7:3, 6:4, 5:5, 4:6, and 3:7) to form five fractions. Each fraction was analyzed by HPLC and dissolved in $\mathrm{MeOH}$ and finally purified by semi-preparative HPLC to give the resulting CRM A, as identified by ${ }^{1} \mathrm{H}$, ${ }^{13} \mathrm{C}$ NMR and HR-ESI-MS.

Analytical HPLC of CRM A was performed on the Agilent 1260 Infinity System with an Agilent Zorbax SB-C18 column $(150 \times 4.6 \mathrm{~mm}, 5 \mu \mathrm{m})$ eluted with a linear gradient of $0 \%$ to $80 \%$ solvent B over 20 min, followed by $80 \%$ to $100 \%$ solvent $\mathrm{B}$ in $30 \mathrm{~s}$, and then eluted with $100 \%$ solvent B in $4.5 \mathrm{~min}$, at a flow rate of $1.0 \mathrm{~mL} / \mathrm{min}$ using UV detection at both $210 \mathrm{~nm}$ and $254 \mathrm{~nm}$. Solvent A was composed of $15 \% \mathrm{CH}_{3} \mathrm{CN}, 85 \% \mathrm{ddH}_{2} \mathrm{O}$, and $0.1 \%$ acetic acid, and solvent $\mathrm{B}$ composed of $85 \% \mathrm{CH}_{3} \mathrm{CN}, 15 \%$ $\mathrm{dd}_{2} \mathrm{O}$, and $0.1 \%$ acetic acid.

To measure the strain's CRM A production titer in the confidence interval, the corresponding quantitative HPLC standard curve was generated by analyzing an authentic CRM A concentration gradient (from 2 to $10 \mu \mathrm{g}$, with $2 \mu \mathrm{g}$ increments). The UV absorption of each analysis was maintained below $1 \mathrm{~A}$ unit to ensure appropriate confidence of the generated standard curve. The strain's fermentation broth $(50 \mathrm{~mL}$ in a $250 \mathrm{~mL}$ flask) was extracted by butanone and concentrated in vacuo to afford the oily residues. These residues were dissolved in $2 \mathrm{~mL} \mathrm{MeOH}$. Then a suitable volume of generated methanol solution was subjected to HPLC analysis to acquire the peak area value. Finally, the titer of different strains was calculated, using a computational formula derived from a standard curve and the volume ratio (Additional file 1: Fig. S6a).

\section{Gene expression analysis of mutant strains}

Total RNA of each mutant was extracted using the SV total RNA purification Kit (Promega) and digested by DNase I (Takara). First-strand cDNA synthesis was accomplished using Invitrogen's SuperScript ${ }^{\mathrm{TM}}$ Kit, and second step PCR was carried out under the following conditions: $94{ }^{\circ} \mathrm{C}$ for $5 \mathrm{~min}, 25$ cycles of denaturation $\left(94{ }^{\circ} \mathrm{C}\right.$ for $25 \mathrm{~s})$, annealing $\left(60{ }^{\circ} \mathrm{C}\right.$ for $\left.25 \mathrm{~s}\right)$, and extension $\left(72{ }^{\circ} \mathrm{C}\right.$ for $45 \mathrm{~s}$ ), and a single extension at $72{ }^{\circ} \mathrm{C}$ for $10 \mathrm{~min}$. A negative control was accordingly performed in the absence of template to check for DNA contamination after the DNase I digestion required for RNA purification.

Quantitative real-time reverse transcription PCR (qPCR) was performed using the Maxima ${ }^{\mathrm{TM}}$ SYBR Green qPCR Mix (MBI) and Applied Biosystem's 7500 Fast Real-time PCR system. 16S rDNA was used as the internal control. The primers used to analyze the camE gene (NCBI accession number: WP_103055331.1) and 16S rDNA are shown in and Additional file 1: Table S17.

\section{UV mutagenesis and biological assay screening of mutant strains}

Diluted spores of selected ribosome engineering mutant strains were put into an uncovered petri dish, which was placed on a magnetic stirrer beneath a UV light on a clean bench. These spores were treated for different irradiation times (5-10 min, with 1 min increments). The preliminary experiments determined that 8 min UV irradiation time confer about $99 \%$ lethal rate and was suitable for strains' mutagenesis operation. After UV irradiation, all strains' spores were spread onto ISP2 plates for subsequent dark cultivation at $28^{\circ} \mathrm{C}$ for 5 days to obtain recovered viable colonies.

The surviving mutant strains were inoculated into test tubes containing $5 \mathrm{~mL}$ ISP2 medium and fermented at $28{ }^{\circ} \mathrm{C}$ for 3 days. Then, the fermentation product was extracted with butanone three times by the ultrasonic treatment. The obtained fermentation extract was dissolved in $50 \mu \mathrm{L}$ methanol. Approximately $10 \mu \mathrm{L}$ liquid extract was dropped onto punched $6 \mathrm{~mm}$ filter papers, which were attached on the LB plates inoculated with not yet grown Escherichia coli ATCC 25922. The colonies 
with the largest inhibition zones were screened for subsequent fermentation verification.

\section{Riboflavin biosynthesis enhancement of mutant strains}

The essential riboflavin biosynthetic gene AsribA (NCBI accession number AUS77540.1), encoding the rate-limiting type II guanosine triphosphate (GTP) cyclohydrolase (GCH II), was completely synthesized (BGI-Shenzhen) and subsequently cloned into the pCR 2.1 vector. The target gene region was digested with $\mathrm{NdeI}$ and $\mathrm{XbaI}$ and ligated into the same digested pSET152AKE to obtain the desired plasmid pSET152AKE-AsribA [48-50]. This constructed plasmid was transformed into E. coli ET12567/ pUZ8002 to construct the donor strain E. coli ET12567/ pUZ8002/pSET152AKE-AsribA, which was then cultivated in $100 \mathrm{~mL} \mathrm{LB}$ with kanamycin (Kan, $50 \mu \mathrm{g} / \mathrm{mL}$ ), chloramphenicol (Chlo, $25 \mu \mathrm{g} / \mathrm{mL}$ ) and apramycin (Apm $50 \mu \mathrm{g} / \mathrm{mL}$ ) at $37^{\circ} \mathrm{C}$ and $200 \mathrm{rpm}$ for $4-6 \mathrm{~h}$ to an OD of 0.6-0.8. Cells were harvested and washed with liquid LB medium, and resuspended in fresh LB medium $(1 \mathrm{~mL})$. The harvested spores of the screened mutant strain were inoculated into $50 \mathrm{~mL}$ liquid ISP2 medium, heated for $10 \mathrm{~min}$ at $50{ }^{\circ} \mathrm{C}$, cooled to ferment at $28{ }^{\circ} \mathrm{C}$ for $6-8 \mathrm{~h}$ and then mixed with the above-harvested $E$. coli ET12567/pUZ8002/pSET152AKE-AsribA cells. The mixed sample was spread on ISP2 plates supplemented with $\mathrm{MgCl}_{2}(20 \mathrm{mM})$. The plates were incubated at $28^{\circ} \mathrm{C}$ for $20 \mathrm{~h}$. Then, these plates were covered with sterile water $(1 \mathrm{~mL})$ supplemented with $30 \mu \mathrm{L}$ trimethoprim (TMP, stock solution $50 \mathrm{mg} / \mathrm{mL}$ ) and $30 \mu \mathrm{L}$ Kan (stock solution $50 \mathrm{mg} / \mathrm{mL}$ ). The plates were incubated at $28{ }^{\circ} \mathrm{C}$ until exconjugants appeared. The exconjugants were verified by PCR using the amplified primers (asribA-Fr: 5'-ACG ACG GTG GAG AGC AGG ACG-3' and asribARe: 5'-TTA TGC CGT CAC TCC CGT TCC-3') to screen the desired gene over-expression mutant.

Analytical HPLC of riboflavin was also performed on Agilent 1260 Infinity System with an Agilent Zorbax SB-C18 column $(150 \times 4.6 \mathrm{~mm}, 5 \mu \mathrm{m})$ eluted as follow: a linear gradient of $0 \%$ to $70 \%$ solvent B over 20 min, followed by $70 \%$ to $100 \%$ solvent B within $15 \mathrm{~s}$, and then eluted with $100 \%$ solvent B within $5 \mathrm{~min}$, at a flow rate of $1.0 \mathrm{~mL} / \mathrm{min}$ with UV detection at $254 \mathrm{~nm}$ and $275 \mathrm{~nm}$. Solvent A was composed of $15 \% \mathrm{CH}_{3} \mathrm{CN}, 85 \% \mathrm{ddH}_{2} \mathrm{O}$, and $0.1 \%$ acetic acid, and solvent $\mathrm{B}$ was composed of $85 \%$ $\mathrm{CH}_{3} \mathrm{CN}, 15 \% \mathrm{ddH}_{2} \mathrm{O}$, and $0.1 \%$ acetic acid. The standard sample of riboflavin was isolated in a previous study [44].

The quantitative HPLC standard curve for comparing riboflavin production titer was generated by analyzing an authentic riboflavin concentration gradient (from $0.2 \mu \mathrm{g}$ to $1 \mu \mathrm{g}$, with $0.2 \mu \mathrm{g}$ increments). The UV absorption of each analysis was maintained below $1 \mathrm{~A}$ unit to ensure appropriate confidence of the generated standard curve. The strain's fermentation broth $(50 \mathrm{~mL}$ in a $250 \mathrm{~mL}$ flask $)$ was extracted by butanone and concentrated in vacuo to afford oily residues. These residues were dissolved in $0.5 \mathrm{~mL} \mathrm{MeOH}$. Then $10 \mu \mathrm{L}$ of generated methanol solution were subjected to HPLC analysis. Finally, the titers of different strains were calculated using a computational formula derived from a standard curve and the volume ratio (Additional file 1: Fig. S6b).

\section{Medium optimization using response surface methodology}

The experiments for optimizing the CRM A production medium were designed and evaluated by using the Minitab 17 software. This software can be used in analyzing the statistical experiment data and response surface contour plots to solve the obtained regression equation, which can finally determine the optimal composition of the fermentation medium.

Preliminary identification of significant variables from CRM A production medium was performed by using Plackett-Burman design. The importance of variables was investigated at widely spaced intervals distinguished as low level $(-1)$ and high level $(+1)$. The effects of each variable on CRM A production were directly calculated in Minitab 17 (with the option "Analyze Factorial Design") by the following equation: E represents the effects of the variable under study, and $\mathrm{M}^{+}$and $\mathrm{M}^{-}$ are responses (CRM A production titer) of each trial at which the variable was as its high or low level, respectively; $\mathrm{N}$ is the total number of trials. Furthermore, the $t$-values and $p$-values of each variable were calculated in Minitab 17 by analyzing factorial design, which was synchronized with the generation of a Pareto chart.

$$
\mathrm{E}=\left(\sum \mathrm{M}^{+}-\sum \mathrm{M}^{-}\right) / \mathrm{N}
$$

Box-Behnken design could accurately analyze the interaction effects among various ingredients and determine the optimal level of significant factors during CRM A production. In this study, an experimental design consisting of three independent variables at three different levels $(-1,0,1)$ was performed in 15 trials. All trials were repeated in triplicate and the average of CRM A titer was set as response $(Y)$. The second order polynomial coefficients were calculated and analyzed using Minitab 17. The general form of the second degree polynomial equation is:

$$
Y=\beta_{0}+\sum \beta_{i} X_{i}+\sum \beta_{i i} X_{i}^{2}+\sum \beta_{i j} X_{i} X_{j}
$$

$Y$ is the predicted response; $X_{i}$ and $X_{j}$ are input variables that influence the response $Y$; $\beta_{0}$ is the offset term; $\beta_{i}$ is the $i$ th linear coefficient; $\beta_{i i}$ is the $i$ th quadratic coefficient; and $\beta_{i j}$ is the $i j$ th interaction coefficient. 
Statistical analysis of the obtained model was performed in ANOVA form. This analysis included the Fisher's $F$-test (overall model significance), its associated probability $P(F)$, correlation coefficient $\mathrm{R}$, and determination coefficient $R^{2}$ which measures the goodness of fit of the regression model. For the variables, the quadratic models were displayed as contour plots and response surface curves which were generated using Minitab 17.

\section{Supplementary information}

Supplementary information accompanies this paper at https://doi. org/10.1186/s12934-020-01418-w.

Additional file 1: Additional file 1: Table S1. Genome features of Actinoalloteichus sp. AHMU CJ021; Table S2. Number of genes associated with the general COG functional categories; Table S3. The biosynthetic gene clusters of secondary metabolites in Actinoalloteichus sp. AHMU CJ021 analyzed by antiSMASH 5.0; Table S4. Deduced functions of the open reading frames (ORFs) indicated in Fig. 2; Table S5. Fermentation media used for caerulomycin A (CRM A) production; Table S6. The mutants obtained from ribosome engineering experiments; Table S7. The CRM A production comparison of three camE-expressing mutants; Table S8. ${ }^{1} \mathrm{H}$ NMR and ${ }^{13} \mathrm{C}$ NMR spectral data of CRM A in DMSO-d6; Table S9. The comparison of selected mutants generated from UV mutagenesis; Table S10. CRM A production titer of optimal mutant XC-11GUR; Table S11. The dose of all factors in medium N2 by using Plackett-Burman Design; Table S12. Screening of significant variables for CRM A production in Medium N2 by using Plackett-Burman Design; Table S13. The effects of all factors of Medium N2 for CRM A production by using Plackett-Burman Design; Table S14. The dose of important factors in response surface analysis; Table S15. The design of experiments and response of CRM A production; Table S16. The primers used in identification of gentamycin-resistant mutant; Table S17. The primers used in gene expression analysis; Fig. S1. Phylogenetic tree of Actinoalloteichus sp. AHMU CJ021; Fig. S2. HR-ESI-MS spectrum of CRM A; Fig. S3. ' H NMR spectrum of CRM A in DMSO-d6; Fig. S4. ${ }^{13} \mathrm{C}$ NMR spectrum of CRM A in DMSO-d6; Fig. S5. CRM A production comparison of different generations of mutants; Fig. S6. The quantitative HPLC standard curves.

\section{Abbreviations}

BGC: Biosynthetic gene cluster; WT: Wild type; RT-PCR: Reverse transcription PCR; qPCR: Quantitative real-time PCR.

\section{Acknowledgements}

We thank the Genome Synthesis and Editing Platform of the China National GeneBank for providing support on gene synthesis. We also acknowledge Shanghai BIOZERON Biotechnology Co., Ltd. for providing support on sequencing and data analysis. We thanks the Springer Nature Author Services for providing support in language editing.

\section{Authors' contributions}

$Y X, X L$ and QC conceived and designed this research. YX, JC, BW and JC conducted experiment. YX, TC, YZ, XL and QC analyzed data. YX and QC wrote the manuscript. All authors read and approved the manuscript.

\section{Funding}

This work was financially supported by NSFC (Grant Nos. 41806158, 41406195, 31760160), by Provincial Natural Science Research Project of Anhui Colleges and Universities (Grant No. KJ2017A177), by the Open Project Program of Guangdong Key Laboratory of Marine Materia Medica \& Key Laboratory of Tropical Marine Bio-resources and Ecology, SCSIO, CAS (Grant No. 2018011007), by the Guangdong Provincial Key Laboratory of Genome Read and Write (No. 2017B030301011), by the Shenzhen Engineering Laboratory for Innovative Molecular Diagnostics (DRC-SZ[2016]884), by the Guangdong Provincial Academician Workstation of BGI Synthetic Genomics (No.
2017B090904014), by the Open Project Program of Key Laboratory of Functional Small Organic Molecule, Ministry of Education, Jiangxi Normal University (No. KLFS-KF-201918).

\section{Availability of data and materials}

All data generated or analyzed during this study are included in this published article and in its additional file.

\section{Ethics approval and consent to participate}

Not applicable.

\section{Consent for publication}

The authors are consent for publication.

\section{Competing interests}

The authors declare that they have no competing interests.

\begin{abstract}
Author details
${ }^{1}$ Key Laboratory of Functional Small Organic Molecule Ministry of Education and Jiangxi's Key Laboratory of Green Chemistry, Key Laboratory of Protection and Utilization of Subtropic Plant Resources of Jiangxi Province, College of Life Sciences, Jiangxi Normal University, Nanchang 330022, China. ${ }^{2}$ School of Life Sciences, Anhui Medical University, Hefei 230032, China. ${ }^{3}$ Guangdong Provincial Key Laboratory of Genome Read and Write, Shenzhen Engineering Laboratory for Innovative Molecular Diagnostics, Guangdong Provincial Academician Workstation of BGI Synthetic Genomics, BGI-Shenzhen, Beishan Industrial Zone, Shenzhen 518083, China. ${ }^{4}$ China National GeneBank, BGI-Shenzhen, Jinsha Road, Shenzhen 518120, China.
\end{abstract}

Received: 3 May 2020 Accepted: 30 July 2020

Published online: 06 August 2020

\section{References}

1. Barka EA, Vatsa P, Sanchez L, Gaveau-Vaillant N, Jacquard C, MeierKolthoff JP, Klenk HP, Clement C, Ouhdouch Y, van Wezel GP. Taxonomy, physiology, and natural products of Actinobacteria. Microbiol Mol Biol R. 2016;80(1):1-43.

2. Durand GA, Raoult D, Dubourg G. Antibiotic discovery: history, methods and perspectives. Int J Antimicrob Agents. 2019;53(4):371-82.

3. Genilloud O. Actinomycetes: still a source of novel antibiotics. Nat Prod Rep. 2017;34(10):1203-32.

4. Hug JJ, Bader CD, Remskar M, Cirnski K, Muller R. Concepts and methods to access novel antibiotics from Actinomycetes. Antibiotics (Basel). 2018;7(2):44.

5. Liu R, Deng ZX, Liu TG. Streptomyces species: ideal chassis for natural product discovery and overproduction. Metab Eng. 2018;50:74-84.

6. Fenical W, Jensen PR. Developing a new resource for drug discovery: marine actinomycete bacteria. Nat Chem Biol. 2006;2(12):666-73.

7. Subramani R, Aalbersberg W. Marine actinomycetes: an ongoing source of novel bioactive metabolites. Microbiol Res. 2012;167(10):571-80.

8. Romano S, Jackson SA, Patry S, Dobson ADW. Extending the "one strain many compounds" (OSMAC) principle to marine microorganisms. Mar Drugs. 2018;16(7):244

9. ul Hassan SS, Shaikh AL. Marine actinobacteria as a drug treasure house. Biomed Pharmacother. 2017:87:46-57.

10. Baral B, Akhgari A, Metsa-Ketela M. Activation of microbial secondary metabolic pathways: avenues and challenges. Synth Syst Biotechnol. 2018;3(3):163-78.

11. Rutledge PJ, Challis GL. Discovery of microbial natural products by activation of silent biosynthetic gene clusters. Nat Rev Microbiol. 2015;13(8):509-23.

12. Ochi K. From microbial differentiation to ribosome engineering. Biosci Biotech Bioch. 2007;71(6):1373-86.

13. Foulston L. Genome mining and prospects for antibiotic discovery. Curr Opin Microbiol. 2019;51:1-8.

14. Hosaka T, Ohnishi-Kameyama M, Muramatsu H, Murakami K, Tsurumi Y, Kodani S, Yoshida M, Fujie A, Ochi K. Antibacterial discovery in 
actinomycetes strains with mutations in RNA polymerase or ribosomal protein S12. Nat Biotechnol. 2009;27(5):462-4.

15. Mao DN, Okada BK, Wu YH, Xu F, Seyedsayamdost MR. Recent advances in activating silent biosynthetic gene clusters in bacteria. Curr Opin Microbiol. 2018;45:156-63.

16. Ochi K, Hosaka T. New strategies for drug discovery: activation of silent or weakly expressed microbial gene clusters. Appl Microbiol Biotechnol. 2013;97(1):87-98.

17. Ochi K, Okamoto S, Tozawa Y, Inaoka T, Hosaka T, Xu J, Kurosawa K. Ribosome engineering and secondary metabolite production. Adv Appl Microbiol. 2004;56:155-84.

18. Zarins-Tutt JS, Barberi TT, Gao H, Mearns-Spragg A, Zhang LX, Newman DJ, Goss RJM. Prospecting for new bacterial metabolites: a glossary of approaches for inducing, activating and upregulating the biosynthesis of bacterial cryptic or silent natural products. Nat Prod Rep. 2016;33(1):54-72.

19. Fu P, Jamison M, La S, MacMillan JB. Inducamides A-C, chlorinated alkaloids from an RNA polymerase mutant strain of Streptomyces sp. Org Lett. 2014;16(21):5656-9.

20. Zhang Y, Huang H, Xu S, Wang B, Ju J, Tan H, Li W. Activation and enhancement of Fredericamycin A production in deepsea-derived Streptomyces somaliensis SCSIO ZH66 by using ribosome engineering and response surface methodology. Microb Cell Fact. 2015;14:64.

21. Fu P, Wang SX, Hong K, Li X, Liu PP, Wang Y, Zhu WM. Cytotoxic bipyridines from the marine-derived actinomycete Actinoalloteichus cyanogriseus WH1-2216-6. J Nat Prod. 2011;74(8):1751-6.

22. Chen M, Zhang YP, Du YN, Zhao QF, Zhang QL, Wu JQ, Liu W. Enzymatic competition and cooperation branch the caerulomycin biosynthetic pathway toward different 2,2'-bipyridine members. Org Biomol Chem. 2017;15(26):5472-5.

23. Zhu YG, Fu P, Lin QH, Zhang GT, Zhang HB, Li SM, Ju JH, Zhu WM, Zhang CS. Identification of caerulomycin A gene cluster implicates a tailoring amidohydrolase. Org Lett. 2012;14(11):2666-9.

24. Chen M, Pang B, Du YN, Zhang YP, Liu W. Characterization of the metallodependent amidohydrolases responsible for "auxiliary" leucinyl removal in the biosynthesis of 2,2'-bipyridine antibiotics. Synth Syst Biotechnol. 2017;2(2):137-46.

25. Garcia I, Vior NM, Brana AF, Gonzalez-Sabin J, Rohr J, Moris F, Mendez C, Salas JA. Elucidating the biosynthetic pathway for the polyketidenonribosomal peptide collismycin A: mechanism for formation of the 2,2'-bipyridyl Ring. Chem Biol. 2012;19(3):399-413.

26. Qu XD, Pang B, Zhang ZC, Chen $M$, Wu ZH, Zhao QF, Zhang QL, Wang YY, Liu Y, Liu W. Caerulomycins and collismycins share a common paradigm for 2,2'-bipyridine biosynthesis via an unusual hybrid polyketide-peptide assembly logic. J Am Chem Soc. 2012;134(22):9038-41.

27. Fu P, Zhu YG, Mei XG, Wang Y, Jia HJ, Zhang CS, Zhu WM. Acyclic congeners from Actinoalloteichus cyanogriseus provide insights into cyclic bipyridine glycoside formation. Org Lett. 2014;16(16):4264-7.

28. Zhu YG, Zhang QB, Li SM, Lin QH, Fu P, Zhang GT, Zhang HB, Shi R, Zhu WM, Zhang CS. Insights into caerulomycin A biosynthesis: a two-component monooxygenase $\mathrm{CrmH}$-catalyzed oxime formation. J Am Chem Soc. 2013;135(50):18750-3.

29. Zhu YG, Picard ME, Zhang QB, Barma J, Despres XM, Mei XG, Zhang LP, Duvignaud JB, Couture M, Zhu WM, Shi R, Zhang CS. Flavoenzyme CrmKmediated substrate recycling in caerulomycin biosynthesis. Chem Sci. 2016;7(8):4867-74.

30. Zhu YG, Xu JX, Mei XG, Feng Z, Zhang LP, Zhang QB, Zhang GT, Zhu WM, Liu JS, Zhang CS. Biochemical and structural insights into the aminotransferase CrmG in caerulomycin biosynthesis. ACS Chem Biol. 2016;11(4):943-52.

31. Kaur S, Srivastava G, Sharma AN, Jolly RS. Novel immunosuppressive agent caerulomycin A exerts its effect by depleting cellular iron content. Br J Pharmacol. 2015;172(9):2286-99.

32. Kujur W, Gurram RK, Maurya SK, Nadeem S, Chodisetti SB, Khan N, Agrewala JN. Caerulomycin A suppresses the differentiation of naive T cells and alleviates the symptoms of experimental autoimmune encephalomyelitis. Autoimmunity. 2017;50(5):317-28.

33. Singla AK, Gurram RK, Chauhan A, Khatri N, Vohra RM, Jolly RS, Agrewala JN. Caerulomycin A: a potent novel immunosuppressive agent. Transplantation. 2014;97(9):e57-9.
34. Shentu X, Liu N, Tang G, Tanaka Y, Ochi K, Xu J, Yu X. Improved antibiotic production and silent gene activation in Streptomyces diastatochromogenes by ribosome engineering. J Antibiot. 2016;69(5):406-10.

35. Tanaka Y, Kasahara K, Izawa M, Ochi K. Applicability of ribosome engineering to vitamin B12 production by Propionibacterium shermanii. Biosci Biotechnol Biochem. 2017;81 (8):1636-41.

36. Wang L, Chen XS, Wu GY, Li S, Zeng X, Ren XD, Tang L, Mao ZG. Enhanced epsilon-poly-L-lysine production by inducing double antibioticresistant mutations in Streptomyces albulus. Bioprocess Biosyst Eng. 2017:40(2):271-83.

37. Zhu XC, Kong JQ, Yang H, Huang $R$, Huang Y, Yang D, Shen B, Duan YW. Strain improvement by combined UV mutagenesis and ribosome engineering and subsequent fermentation optimization for enhanced 6'-deoxy-bleomycin Z production. Appl Microbiol Biotechnol. 2018;102(4):1651-61.

38. Wang M, Chen B, Fang Y, Tan T. Cofactor engineering for more efficient production of chemicals and biofuels. Biotechnol Adv. 2017;35(8):1032-9.

39. Chen R, Yang S, Zhang L, Zhou YJ. Advanced strategies for production of natural products in yeast. iscience. 2020;23(3):100879.

40. Liu J, Li H, Zhao G, Caiyin Q, Qiao J. Redox cofactor engineering in industrial microorganisms: strategies, recent applications and future directions. J Ind Microbiol Biotechnol. 2018:45(5):313-27.

41. Li X, Chen J, Andersen JM, Chu J, Jensen PR. Cofactor engineering redirects secondary metabolism and enhances erythromycin production in Saccharopolyspora erythraea. ACS Synth Biol. 2020;9(3):655-70.

42. Hümbelin M, Griesser V, Keller T, Schurter W, Haiker M, Hohmann H-P, Ritz H, Richter G, Bacher A, van Loon APGM. GTP cyclohydrolase II and 3,4-dihydroxy-2-butanone 4-phosphate synthase are rate-limiting enzymes in riboflavin synthesis of an industrial Bacillus subtilis strain used for riboflavin production. J Ind Microbiol Biotechnol. 1999;22(1):1-7.

43. Birkenmeier M, Neumann S, Röder T. Kinetic modeling of riboflavin biosynthesis in Bacillus subtilis under production conditions. Biotechnol Lett. 2014;36(5):919-28.

44. Chen Q, Wu CF, Zhang Y, Liu XY, Chen JY, Lei L, Xie YC. Enhancement of marinacarboline A biosynthesis by optimizing the riboflavin supplement in marine derived Nocardiopsis flavescens CGMCC 4.5723. Biotechnol Lett. 2019;41(8-9):1033-41.

45. Pfister P, Hobbie S, Vicens Q, Bottger EC, Westhof E. The molecular basis for A-site mutations conferring aminoglycoside resistance: relationship between ribosomal susceptibility and X-ray crystal structures. ChemBioChem. 2003;4(10):1078-88.

46. Vila-Sanjurjo A, Lu Y, Aragonez JL, Starkweather RE, Sasikumar M, O'Connor M. Modulation of $16 \mathrm{~S}$ rRNA function by ribosomal protein S12. Biochim Biophys Acta. 2007;1769(7-8):462-71.

47. Davies C, Bussiere DE, Golden BL, Porter SJ, Ramakrishnan V, White SW. Ribosomal proteins S5 and L6: high-resolution crystal structures and roles in protein synthesis and antibiotic resistance. J Mol Biol. 1998;279(4):873-88.

48. Xie YC, Li QL, Song YX, Ma JY, Ju JH. Involvement of SgvP in carbonsulfur bond formation during griseoviridin biosynthesis. ChemBioChem. 2014;15(8):1183-9.

49. Xie YC, Wang B, Liu J, Zhou JC, Ma JY, Huang HB, Ju JH. Identification of the biosynthetic gene cluster and regulatory cascade for the synergistic antibacterial antibiotics griseoviridin and viridogrisein in Streptomyces griseoviridis. ChemBioChem. 2012;13(18):2745-57.

50. Xie YC, Ma JY, Qin XJ, Li QL, Ju JH. Identification and utilization of two important transporters: SgvT1 and SgvT2, for griseoviridin and viridogrisein biosynthesis in Streptomyces griseoviridis. Microb Cell Fact. 2017;16(1):177.

\section{Publisher's Note}

Springer Nature remains neutral with regard to jurisdictional claims in published maps and institutional affiliations. 